\title{
Implementation of a Boundary Element Method for High Frequency Scattering by Convex Polygons with Impedance Boundary Conditions
}

\author{
Mosiamisi Mokgolele \\ Botswana University of Agriculture and Natural Resourses, Private Bag 0027, Gaborone, Botswana.
}

(Received 13 July 2015; accepted 26 August 2016)

\begin{abstract}
Many acoustic and electromagnetic wave scattering problems can be formulated as the Helmholtz equation. Standard finite and boundary element method solution of these problems becomes expensive, as the frequency of incident wave increases. On going research has been devoted to finding methods that do not loose robustness when the wave number increases. Recently, Chandler-Wilde et al. have proposed a novel Galerkin boundary element method to solve the problem of acoustic scattering by a convex polygon with impedance boundary conditions. They applied approximation spaces consisting of piecewise polynomials supported on a graded mesh with smaller elements adjacent to the corners of the polygon and multiplied by plane wave basis functions. They demonstrated via rigorous error analysis that was supported by numerical experiments that the number of degrees of freedom required to achieve a prescribed level of accuracy need only grow logarithmically as frequency increases. In this paper, we discuss issues related to detail implementation of their numerical method.
\end{abstract}

\section{INTRODUCTION}

We consider the two-dimensional problem of scattering of a time-harmonic acoustic incident plane wave:

$$
u^{i}(\mathbf{x})=\mathrm{e}^{\mathrm{i} k \mathbf{x} \cdot \mathbf{d}}, \text { in } D:=\mathbb{R}^{2} \backslash \bar{\Omega}
$$

by a convex polygon $\Omega$, with impedance boundary $\Gamma$. Here $\mathbf{x}=\left(x_{1}, x_{2}\right) \in \mathbb{R}^{2}, \mathbf{d}=(\sin \theta,-\cos \theta) \in \mathbb{R}^{2}$ is a unit vector representing the direction of the incident field, $\theta$ is the incidence angle, and the frequency of the incident wave is proportional to the wavenumber $k>0$. The scattered field $u^{s}:=u^{t}-u^{i} \in C^{2}(\bar{D})$ (where $u^{t}$ and $u^{i}$ denote the total and incident field respectively) satisfies the Helmholtz equation:

$$
\Delta u^{s}+k^{2} u^{s}=0, \text { in } D .
$$

We shall consider the impedance boundary condition here:

$$
\frac{\partial u^{t}}{\partial \mathbf{n}}+\mathrm{i} k \beta u^{t}=0, \text { on } \Gamma
$$

(where $\mathbf{n}=\left(n_{1}, n_{2}\right)$ denotes the outward unit normal vector to $\Gamma$, as depicted in Fig. 1 and $\beta \in L^{\infty}(\Gamma)$ and $\operatorname{Re} \beta>0$ is relative surface admittance), and is supplemented with the Sommerfeld radiation condition:

$$
\lim _{r \rightarrow \infty} r^{\frac{1}{2}}\left(\frac{\partial u^{s}}{\partial r}-\mathrm{i} k u^{s}\right)=0 ;
$$

where $r:=|\mathbf{x}|$ and the limit holds uniformly in $\mathbf{x} /|\mathbf{x}|$. The Sommerfeld radiation condition is essential to scattering problems because it ensures that the scattered field is not reflected back from infinity.

As $k$ increases, the incident field oscillates more rapidly, and so the complexity of the solution of Eq.(2) increases. As a result, the computational cost of standard schemes, such as the finite element or boundary element methods will grow in direct proportion to $k$, leading to large computing times for large $k$. It has been shown that in order to accurately model a wave, a fixed number of degrees of freedom $M$ are needed per wavelength, with a rule of thumb in the engineering literature of 6 to 10 degrees of freedom per wavelength needed to maintain accuracy. ${ }^{1,2}$ The price to pay for fixing $\mathrm{M}$ is that the number of degrees of freedom will be proportional to $(k L)^{d-1}$ in case of boundary element methods, where $L$ is the linear dimension of the scattering obstacle and $d=2$ or 3 is the dimension of the problem. Thus, as either $k$ or the size of the scatterer grows, so does the number of degrees of freedom (at least $\mathcal{O}(k)$ ) in two-dimensional, hence the computational cost of numerical schemes increases. The previous and the current development on this active field of scattering problems is outlined explicitly at length in. ${ }^{3}$

For this paper we begin in Section 2 by discussing the boundary integral method we are going to apply. We describe the approximation space for the problem in Section 3. We proceed in Section 4 by presenting the implementation of our Galerkin scheme. We present formulas for the Galerkin scheme and describe how to evaluate oscillatory and non oscillatory integrals. In Section 5, we discuss how to solve nonoverlapping integrals, a detail explanation of Gaussian quadrature rule is also explained in this section. In Section 6 we choose an example for our numerical experiment and present some results, whereas most of them can be found in. ${ }^{4,5}$ We discuss our conclusion and some recommendations in Section 7.

\section{BOUNDARY INTEGRAL EQUATION METHOD}

The boundary value problem Eqs. (2) - (4) can be reformulated into boundary integral equation by applying Green's rep- 


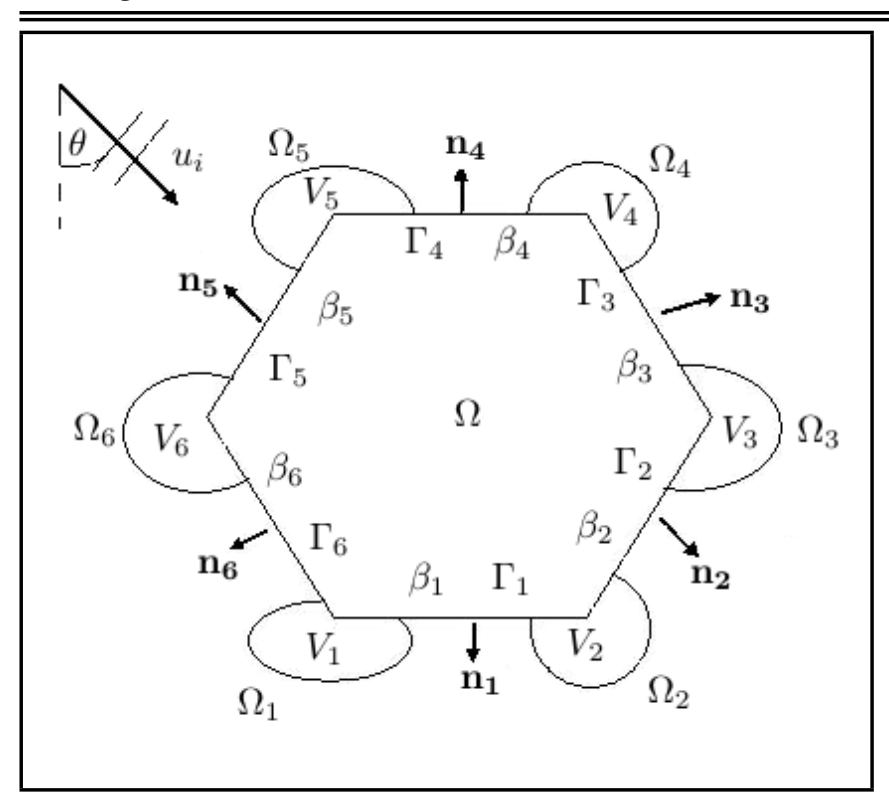

Figure 1. Scattering by an impedance convex polygon.

resentation theorems [7.12 and 9.6 $]^{6}$ and Green's second theorem [theorem 4.4], ${ }^{6}$ (see also ${ }^{4}$ for details), which leads to standard boundary integral equation for unknown $u^{t}$ :

$$
\begin{array}{r}
u^{t}(\mathbf{x})-2 \int_{\Gamma}\left(\frac{\partial \Phi^{\prime}(\mathbf{x}, \mathbf{y})}{\partial \mathbf{n}(\mathbf{y})}+\mathrm{i} k \beta(\mathbf{y}) \Phi^{\prime}(\mathbf{x}, \mathbf{y})\right) u^{t}(\mathbf{y}) \mathrm{d} s(\mathbf{y})= \\
2 f(\mathbf{x}), \quad \mathbf{x} \in \Gamma ; \quad
\end{array}
$$

where $\Phi^{\prime}(\mathbf{x}, \mathbf{y}):=\frac{\mathrm{i}}{4} H_{0}^{(1)}(k|\mathbf{x}-\mathbf{y}|),\left(H_{0}^{(1)}\right.$ is the Hankel function of first kind of order zero) and $f(\mathbf{x})=u^{i}(\mathbf{x})$. The integral Eq. (5) suffers from so-called spurious eigenfrequencies; that is, it is not uniquely solvable for all wavenumbers. ${ }^{7,8}$ However, this problem is often ignored in the literature. ${ }^{7}$ Various options exist to overcome this difficulty. ${ }^{4}$

We begin by defining some notations, see Fig. 1. We write the boundary of the polygon as $\Gamma=\cup_{j=1}^{n_{s}} \Gamma_{j}$, where $\Gamma_{j}, j=$ $1, \ldots, n_{s}$ are the $n_{s}^{t h}$ sides of the polygon, ordered so that $\Gamma_{j}$, $j=1, \ldots, n_{s h}$, are in shadow, and $\Gamma_{j}, j=n_{s h}+1, \ldots, n_{s}$ are illuminated, with $j$ increasing anticlockwise, as shown in Fig. 1.

We denote the corners of the polygon by $V_{j}, j=1, \ldots, n_{s}$, and set $V_{n_{s}+1}=V_{1}$, so that for $j=1, \ldots, n_{s}, \Gamma_{j}$ is the line joining $V_{j}$ with $V_{j+1}$. We denote the length of $\Gamma_{j}$ by $L_{j}=\left|V_{j+1}-V_{j}\right|$, the external angle at each vertex $V_{j}$ by $\Omega_{j} \in(\pi, 2 \pi)$, the unit normal vector to $\Gamma_{j}$ by $\mathbf{n}_{j}$, and the angle of the incident plane wave, as measured anticlockwise from the downward vertical, by $\theta \in[0,2 \pi)$. We represent $\mathbf{x} \in \Gamma$ parametrically by:

$$
\begin{aligned}
& \mathbf{x}(s)=V_{j}+\left(s-\sum_{n=1}^{j-1} L_{n_{s}}\right)\left(\frac{V_{j+1}-V_{j}}{L_{j}}\right) \\
& \quad \text { for } s \in\left(\sum_{n=1}^{j-1} L_{n_{s}}, \sum_{n=1}^{j} L_{n}\right), j=1, \ldots, n_{s} .
\end{aligned}
$$

Define

$$
\begin{aligned}
& \mathbf{x}(s)=\left(x_{1}(s), x_{2}(s)\right)=\left\{\left(e_{l} s+g_{l}\right),\left(f_{l} s+h_{l}\right)\right\} \\
& \mathbf{x}(t)=\left(x_{1}(t), x_{2}(t)\right)=\left\{\left(e_{j} t+g_{j}\right),\left(f_{j} t+h_{j}\right)\right\}
\end{aligned}
$$

where

$$
\begin{aligned}
e_{j} & :=\frac{u_{j+1}-u_{j}}{L_{j}}, \\
f_{j} & :=\frac{v_{j+1}-v_{j}}{L_{j}}, \\
g_{j} & :=u_{j}-e_{j} \sum_{n=1}^{j-1} L_{n}, \\
h_{j} & :=v_{j}-f_{j} \sum_{n=1}^{j-1} L_{n_{s}} ;
\end{aligned}
$$

here point $s$ is in element $l$ and point $t$ is in element $j$. We can write Eq. (5) in parametric form as:

$$
\phi(s)-2 \int_{0}^{L} K(s, t) \phi(t) \mathrm{d} t=2 f(s)
$$

where $\phi(s)=u^{t}(\mathbf{x}(s)), L=\sum_{n=1}^{j} L_{n_{s}}$,

$$
K(s, t):=\left(\frac{\partial \Phi^{\prime}(\mathbf{x}(s), \mathbf{x}(t))}{\partial \mathbf{n}(\mathbf{x}(t))}+\mathrm{i} k \beta(\mathbf{x}(t)) \Phi^{\prime}(\mathbf{x}(s), \mathbf{x}(t))\right)
$$

and $\Phi^{\prime}(\mathbf{x}(s), \mathbf{x}(t)):=\frac{\mathrm{i}}{4} H_{0}^{(1)}(k|\mathbf{x}(s)-\mathbf{x}(t)|)=\frac{\mathrm{i}}{4} H_{0}^{(1)}(k R)$, here

$$
\begin{aligned}
& R= \\
& \sqrt{\left[\left(e_{l} s+g_{l}\right)-\left(e_{j} t+g_{j}\right)\right]^{2}+\left[\left(f_{l} s+h_{l}\right)-\left(g_{j} t+h_{j}\right)\right]^{2}} ;
\end{aligned}
$$

and finally

$$
\begin{gathered}
f(s)=u^{i}(s)=\mathrm{e}^{\mathrm{i} k\left(x_{1}(s) \sin \theta-x_{2}(s) \cos \theta\right)}= \\
\mathrm{e}^{\mathrm{i} k\left(\left(e_{l} s+g_{l}\right) \sin \theta-\left(f_{l} s+h_{l}\right) \cos \theta\right)} .
\end{gathered}
$$

We know that $\left(n_{1 j}, n_{2 j}\right) \cdot\left(e_{l}, f_{l}\right)=0$, which implies $n_{1 j} e_{l}+$ $n_{2 j} f_{l}=0$, which implies $n_{1 j}=f_{l}, n_{2 j}=-e_{l}$ or $n_{1 j}=$ $-f_{l}, n_{2 j}=e_{l}$ (depending on whether the unit normal is inward or outward). Using the fact that $\frac{\partial H_{0}^{(1)}(z)}{\partial z}=-H_{1}^{(1)}(z)$, and also that $\frac{\partial}{\partial \mathbf{n}}=\mathbf{n} \cdot \nabla$, we can now evaluate the explicit formula for:

$$
\begin{aligned}
& \frac{\partial \Phi^{\prime}(\mathbf{x}(s), \mathbf{x}(t))}{\partial \mathbf{n}(\mathbf{x}(t))}= \\
& n_{1 l} \frac{\partial \Phi^{\prime}(\mathbf{x}(s), \mathbf{x}(t))}{\partial x_{1}(t)}+n_{2 l} \frac{\partial \Phi^{\prime}(\mathbf{x}(s), \mathbf{x}(t))}{\partial x_{2}(t)}= \\
& -\frac{\mathrm{i} k}{4} \frac{H_{1}^{(1)}(k R)}{R}\left[f_{j}\left(x_{1}(t)-x_{1}(s)\right)-e_{j}\left(x_{2}(t)-x_{2}(s)\right)\right]= \\
& \quad-\frac{\mathrm{i} k}{4} \frac{H_{1}^{(1)}(k R)}{R} \\
& \cdot\left[\left(e_{j} f_{l}-f_{j} e_{l}\right) s+f_{j}\left(g_{j}-g_{l}\right)+e_{j}\left(h_{l}-h_{j}\right)\right] .
\end{aligned}
$$


Therefore,

$$
\begin{aligned}
K(s, t)= & \\
-\frac{k}{4}\left\{\mathrm{i} \frac{H_{1}^{(1)}(k R)}{R}\right. & \\
{\left[\left(e_{j} f_{l}-f_{j} e_{l}\right) s+f_{j}\left(g_{j}-g_{l}\right)\right.} & \left.+e_{j}\left(h_{l}-h_{j}\right)\right] \\
& \left.+\beta H_{0}^{(1)}(k R)\right\} .
\end{aligned}
$$

We know that on each side of the polygon $u^{t}=u^{i}+u^{r}+u^{d}$, where $u^{r}$ is the field that would be reflected by a side $\Gamma_{j}$, if that side was infinitely long, and $u^{d}$ represents in some sense the waves diffracted by the corners of the polygon . ${ }^{9}$ For a straight line polygon, we know $u^{r}$ explicitly, and particularly for a straight line polygon with an impedance bound-

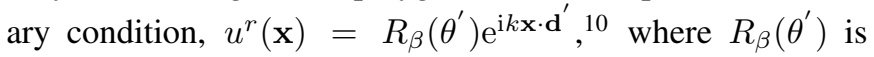
the reflection coefficient that is given by a reflective angle, $R_{\beta}\left(\theta^{\prime}\right)=\left(\frac{\cos \theta^{\prime}-\beta}{\cos \theta^{\prime}+\beta}\right), \theta^{\prime}$ which depends on $\theta$ and $\Gamma_{j}$ and $\mathbf{d}^{\prime}=(\sin \theta, \cos \theta)$. Since $u^{r}$ is a function of the incident angle, it is only featured on the illuminated side and it is zero on the shadow side of the polygon. We define our leading order behavior:

$$
\begin{aligned}
\Psi(s) & :=\left\{\begin{array}{r}
u^{i}(s)+u^{r}(s), \text { in illuminated region, } \\
0, \text { in shadow region, }
\end{array}\right. \\
& =\left\{\begin{array}{c}
2 \frac{\cos \theta^{\prime}}{\cos \theta^{\prime}+\beta} u^{i}(s), \text { in illuminated region, } \\
0, \text { in shadow region, }
\end{array}\right.
\end{aligned}
$$

and $\Phi(s):=u^{d}(s)$ is viewed as the diffracted wave due to the corner of the polygon. Therefore, subtracting the leading order behavior gives:

$$
\Phi(s):=\phi(s)-\Psi(s)
$$

Substituting Eq. (16) into Eq. (9), we obtain a new second kind boundary integral equation with unknown $\Phi(s)$ :

$$
(I-\mathcal{K}) \Phi(s)=F(s)
$$

where $\mathcal{K} v(s):=2 \int_{0}^{L} K(s, t) v(t) \mathrm{d} t, F(s):=2 f(s)-\Psi(s)+$ $2 \int_{0}^{L} K(s, t) \Psi(t) \mathrm{d} t$, and $I$ is the identity operator.

\section{APPROXIMATION SPACE}

We begin by defining a general mesh grading on $\left[0, L_{j}\right]$, which is composed of a polynomial grading on $[0, \lambda]$ and a geometric grading on $\left[\lambda, L_{j}\right]$, where $L_{j}$ is the length of the $j^{t h}$ side, $j=1 \ldots, n_{s}$ (recalling that $n_{s}$ is the number of sides of a polygon) $\lambda=2 \pi / k$ is the wavelength. We now define our mesh as follows: for $L_{j}>\lambda>0, q_{j}>0, N=2,3, \ldots$, where $q_{j}=\frac{2 \nu+3}{2 \pi / \Omega_{j}+1}$ and $\nu$ is degree of a polynomial. The mesh

$\Lambda_{N, L_{j}, \lambda, q_{j}}:=\left\{y_{0}, \ldots, y_{N+N_{L_{j}, \lambda, q_{j}}}\right\}$ consists of the points:

$$
\begin{gathered}
y_{i}=\lambda\left(\frac{i}{N}\right)^{q_{j}}, \quad i=0, \ldots, N \\
y_{N+j}:=\lambda\left(\frac{L_{j}}{\lambda}\right)^{j / N_{L_{j}, \lambda, q_{j}}}, \quad j=1, \ldots, N_{L_{j}, \lambda, q_{j}} .
\end{gathered}
$$

Where $N_{L_{j}, \lambda, q_{j}}=\left\lceil\hat{N}_{L_{j}, \lambda, q_{j}}\right\rceil$,

$$
\hat{N}_{L_{j}, \lambda, q_{j}}=\frac{-\log \left(L_{j} / \lambda\right)}{q_{j} \log (1-1 / N)} .
$$

Here, $\lceil z\rceil$ denotes the smallest integer greater than or equal to $z$, for $z \in \mathbb{R}$. Specifically, $N_{L_{j}, \lambda, q_{j}}$ is the smallest positive integer greater or equal to Eq. (20).

The mesh we propose is that away from the corner (between $\left.\left[\lambda, L_{j}\right]\right)$ the mesh is chosen such that it is independent of $q_{j}$, while near a corner (between $[0, \lambda]$ ) the mesh is chosen such that it is independent of $L_{j}$. It is reasonable to choose the mesh such that for a fixed $N_{1}$ :

$$
\left.N=\mathcal{O}\left(N_{1} q_{j}\right) \text {, between }[0, \lambda]\right) .
$$

We now take $N$, greater or equal to $\hat{c} N_{1} q_{j}$, and use Eq. (20) to compute $N_{L_{j}, \lambda, q_{j}}$, where here $\hat{c}$ is an arbitrary constant. We now define the two meshes:

$$
X_{j}:=\tilde{L}_{j-1}+\Lambda_{N, L_{j}, \lambda, q_{j}}, \quad Y_{j}:=\tilde{L}_{j}-\Lambda_{N, L_{j}, \lambda, q_{j+1}} .
$$

This choice of $q_{j}$ ensures that the approximation error is evenly spread on each mesh interval. Letting $\mathrm{e}_{ \pm}(s):=\mathrm{e}^{ \pm \mathrm{i} k s}, s \in$ $\left[0, L_{j}\right]$, we then define the approximation spaces associated with each mesh as:

$$
\begin{aligned}
A_{X_{j}, \nu} & :=\left\{\sigma \mathrm{e}_{+}: \sigma \in \Pi_{X_{j}, \nu}\right\}, \\
A_{Y_{j}, \nu} & :=\left\{\sigma \mathrm{e}_{-}: \sigma \in \Pi_{Y_{j}, \nu}\right\}
\end{aligned}
$$

for $j=1, \ldots, n_{s}$, where

$$
\begin{gathered}
\Pi_{X_{j}, \nu}:=\left\{\sigma \in L^{2}\left(0, L_{n_{s}}\right):\left.\sigma\right|_{\left(\tilde{L}_{j-1}+x_{m-1}, \tilde{L}_{j-1}+x_{m}\right)}\right. \\
\text { is a polynomial of degree } \leq \nu, \\
\quad \text { for } m=1, \ldots, N+N_{L_{j}, \lambda, q_{j}}, \\
\left.\quad \text { and }\left.\sigma\right|_{\left(0, \tilde{L}_{j-1}\right) \cup\left(\tilde{L}_{j}, L\right)}=0\right\}, \\
\Pi_{Y_{j}, \nu}:=\left\{\sigma \in L^{2}\left(0, L_{n_{s}}\right):\left.\sigma\right|_{\left(\tilde{L}_{j}-y_{m}, \tilde{L}_{j}-y_{m-1}\right)}\right. \\
\text { is a polynomial of degree } \leq \nu, \\
\text { for } m=1, \ldots, N+N_{L_{j}, \lambda, q_{j+1}}, \\
\text { and } \left.\left.\sigma\right|_{\left(0, \tilde{L}_{j-1}\right) \cup\left(\tilde{L}_{j}, L\right)}=0\right\} ;
\end{gathered}
$$

where $\left\{x_{0}, \ldots, x_{N+N_{L_{j}, \lambda, q_{j}}}\right\}$ and $\left\{y_{0}, \ldots, y_{N+N_{L_{j}, \lambda, q_{j+1}}}\right\}$ denote the points of the meshes $\Lambda_{N, L_{j}, \lambda, q_{j}}$ and $\Lambda_{N, L_{j}, \lambda, q_{j+1}}$ respectively. Our approximation space $A_{N, \nu}$ is then the linear span of

$$
\bigcup_{j=1, \ldots, n_{s}}\left\{A_{X_{j}, \nu} \cup A_{Y_{j}, \nu}\right\} .
$$

The number of the degrees of freedom for this problem will be:

$$
D_{N}=2(\nu+1) \sum_{j=1}^{n_{s}}\left(N+N_{L_{j}, \lambda, q_{j}}\right) ;
$$

where the number 2 on the left hand side is due to the fact that we have two meshes Eq. (22), $N$ is the number of points between $[0, \lambda]$ and $N_{L_{j}, \lambda, q_{j}}$ is the number of points between $\left[\lambda, L_{j}\right]$. For our Galerkin Scheme in this paper, we take $\nu=0$. 
Equation (17) is the one we are going to solve for the unknown $\Phi(s)$ by the Galerkin boundary element method. As our starting point we seek:

$$
\Phi(s) \approx \Phi_{N}(s):=\sum_{m=1}^{D_{N}} v_{m} \eta_{m}(s)
$$

where $v_{m}$ is the unknown coefficient, $\eta_{m}$ are the basis functions, and $D_{N}$ is the total number of basis functions. For $j=1, \ldots, n_{s}$, we define $n_{j}^{+}$and $n_{j}^{-}$to be the number of points of the two meshes $X_{j}$ and $Y_{j}$ respectively, so

$$
n_{j}^{+}:=N+N_{L_{j}, \lambda, q_{j}}, \quad n_{j}^{-}:=N+N_{L_{j}, \lambda, q_{j+1}} .
$$

We denote the number of points of $X_{j}$ and $Y_{j}$ by $s_{j, l}^{+}$and $s_{j, l}^{-}$ respectively, for $j=1, \ldots, n_{s}, l=1, \ldots, n_{j}^{ \pm}$. We denote the total number of elements supported on $\cup_{i=1}^{j} \Gamma_{i}$ by

$$
D_{N, j}:=\sum_{i=1}^{j}\left(n_{i}^{+}+n_{i}^{-}\right)
$$

so that the total number of degrees of freedom is $D_{N}=D_{N, j}$, that is,

$$
D_{N}:=\sum_{j=1}^{n}\left(n_{j}^{+}+n_{j}^{-}\right)
$$

Then, for $j=1, \ldots, n$, the basis functions are given by

$$
\begin{gathered}
\eta_{D_{N, j-1}+j}(s):=\frac{\mathrm{e}^{\mathrm{i} k s}}{\sqrt{s_{j, l}^{+}-s_{j, l-1}^{+}}} \chi_{\left[s_{j, l-1}^{+}, s_{j, l}^{+}\right)}(s), \\
j=1, \ldots, n_{j}^{+}, \\
\eta_{D_{N, j-1}+n_{j}^{+}+l}(s):=\frac{\mathrm{e}^{-\mathrm{i} k s}}{\sqrt{s_{j, l}^{-}-s_{j, l-1}^{-}}} \chi_{\left[s_{j, l-1}^{-}, s_{j, l}^{-}\right)}(s), \\
\quad j=1, \ldots, n_{j}^{-} ;
\end{gathered}
$$

where $\chi_{\left[z_{m}, z_{m+1}\right)}$ denotes the characteristic function of the interval $\left[z_{m}, z_{m+1}\right)\left(z_{i}, i=0, \ldots, D_{N}\right.$, are the points of each mesh).

\section{GALERKIN SCHEME}

Substituting Eq. (27) into Eq. (17) and multiplying by a test function $\bar{\eta}_{l}(s)$ and then integrating over $[0, L]$, gives the following Garlekin scheme (which is a system of linear equations):

$$
\sum_{m=1}^{D_{N}}\left[\left(\eta_{m}, \eta_{l}\right)-\left(\mathcal{K} \eta_{m}, \eta_{l}\right)\right] v_{m}=\left(F, \eta_{l}\right) .
$$

Now we have to figure out how to compute $\left(\eta_{m}, \eta_{l}\right),\left(\mathcal{K} \eta_{m}, \eta_{l}\right)$ and $\left(F, \eta_{l}\right)$. To evaluate these matrices, we follow a similar procedure to that in Langdon and Chandler-Wilde, ${ }^{11}$ where most of the formulas are similar. When evaluating the above integrals, we encountered difficulties of dealing with singularities and oscillatory integrals at high frequencies. For the specific problem we are going to discuss here, we encounter the singularities when the basis functions are supported on different side of polygon. When they are supported on the same side of the polygon, there is hardly any singularity. As such, most of integrals can be evaluated analytically. Ideas dealing with oscillatory integrals is on going research. ${ }^{12,13}$ For our problem here, we address the oscillatory integrals by applying standard Gaussian quadrature rule, see Section 5 for detail.

\subsection{Evaluation of $\left(\eta_{m}, \eta_{l}\right)$}

The mass matrix will appear in the following form:

$$
\left(\eta_{m}, \eta_{l}\right)=\int_{\operatorname{supp}\left(\eta_{m}\right) \cap \operatorname{supp}\left(\eta_{l}\right)} \frac{\mathrm{e}^{\left(\delta_{m}-\delta_{l}\right) \mathrm{i} k s}}{\sqrt{\left(z_{m+1}-z_{m}\right)\left(z_{l+1}-z_{l}\right)}} \mathrm{d} s .
$$

This can be evaluated analytically. Here, $\delta_{m}= \pm 1$. Notice that, if $\eta_{m}$ and $\eta_{l}$ are supported on different sides of the polygon, there is no overlap. Hence $\left(\eta_{m}, \eta_{l}\right)=0$. If $\eta_{m}$ and $\eta_{l}$ are supported on the same side of the polygon and $\delta_{m}=\delta_{l}$, then there will be total overlap, this forms the diagonal of the whole matrix $\left(\eta_{m}, \eta_{l}\right)$ and in this case $\left(\eta_{m}, \eta_{l}\right)=1$. If $\eta_{m}$ and $\eta_{l}$ are supported on the same side of the polygon and $\delta_{m} \neq \delta_{l}$, then there will be some overlaps and non-overlaps. In this case, we integrate between the overlapping intervals. If we define the lower and the upper integrating limits respectively as

$$
L_{w}=\min \left(z_{l+1}, z_{m+1}\right) \quad \text { and } \quad U_{p}=\max \left(z_{l}, z_{m}\right) ;
$$

then

$$
\begin{aligned}
&\left(\eta_{m}, \eta_{l}\right)= \int_{L_{w}}^{U_{p}} \eta_{m}(s) \bar{\eta}_{l}(s) \mathrm{d} s= \\
& \frac{\mathrm{e}^{-2 \mathrm{i} k U_{p}}-\mathrm{e}^{-2 \mathrm{i} k L_{w}}}{-2 \mathrm{i} k \sqrt{\left(z_{m+1}-z_{m}\right)\left(z_{l+1}-z_{l}\right)}} \\
& \text { if } \quad \delta_{m}=-1 \quad \text { and } \quad \delta_{l}=1 ;
\end{aligned}
$$

similarly,

$$
\begin{aligned}
&\left(\eta_{m}, \eta_{l}\right)= \int_{L_{w}}^{U_{p}} \eta_{m}(s) \bar{\eta}_{l}(s) \mathrm{d} s= \\
& \frac{\mathrm{e}^{-2 \mathrm{i} k U_{p}}-\mathrm{e}^{-2 \mathrm{i} k L_{w}}}{2 \mathrm{i} k \sqrt{\left(z_{m+1}-z_{m}\right)\left(z_{l+1}-z_{l}\right)}}, \\
& \quad \text { if } \delta_{m}=1 \quad \text { and } \quad \delta_{l}=-1 .
\end{aligned}
$$

\subsection{Evaluation of $\left(\mathcal{K} \eta_{m}, \eta_{l}\right)$}

Evaluating this integral is a challenge because it envolves double integrals. It will appear in the following form:

$$
\left(\mathcal{K} \eta_{m}, \eta_{l}\right)=2 \int_{\operatorname{supp}\left(\eta_{l}\right)} \int_{\operatorname{supp}\left(\eta_{m}\right)} K(s, t) \eta_{m}(t) \bar{\eta}_{l}(s) \mathrm{d} t \mathrm{~d} s .
$$

If we first consider the case where $\eta_{m}$ and $\eta_{l}$ are supported on the same side of the polygon, then we see immediately from Eq. (14) that $e_{l}=e_{j}, f_{l}=f_{j}, g_{l}=g_{j}$, and $h_{l}=h_{j}$, hence the term $H_{1}^{(1)}$ vanishes and Eq. (14) becomes:

$$
K(s, t)=-\frac{k}{4} \beta H_{0}^{(1)}(k|s-t|) .
$$

We know from $[(12.31)]^{14}$ that

$$
H_{0}^{(1)}(s)=\frac{-2 \mathrm{i}}{\pi} \int_{0}^{\infty} \frac{\mathrm{e}^{(\mathrm{i}-t) s}}{t^{\frac{1}{2}}(t-2 \mathrm{i})^{\frac{1}{2}}} \mathrm{~d} t, \quad s>0 ;
$$


SO

$$
\begin{gathered}
\left(\mathcal{K} \eta_{m}, \eta_{l}\right)=-\frac{k \beta}{2} \int_{z_{l}}^{z_{l+1}} \int_{z_{m}}^{z_{m+1}} H_{0}^{1}(k|s-t|) \\
\cdot \frac{\mathrm{e}^{\mathrm{i} k\left(\delta_{m} t-\delta_{l} s\right)}}{\sqrt{\left(z_{l+1}-z_{l}\right)\left(z_{m+1}-z_{m}\right)}} \mathrm{d} t \mathrm{~d} s= \\
\frac{\mathrm{i} k \beta}{\pi \sqrt{\left(z_{l+1}-z_{l}\right)\left(z_{m+1}-z_{m}\right)}} \int_{0}^{\infty} \frac{J(r)}{r^{\frac{1}{2}}(r-2 i)^{\frac{1}{2}}} \mathrm{~d} r
\end{gathered}
$$

where

$$
J(r)=\int_{z_{l}}^{z_{l+1}} \int_{z_{m}}^{z_{m+1}} \mathrm{e}^{-r k|s-t|+\mathrm{i} k\left(|s-t|+\delta_{m} t-\delta_{l} s\right)} \mathrm{d} t \mathrm{~d} s .
$$

We explore further on how to evaluate Eq. (41), depending on how $\left[z_{l}, z_{l+1}\right]$ and $\left[z_{m}, z_{m+1}\right]$ overlap. In the case where $\left[z_{l}, z_{l+1}\right]$ and $\left[z_{m}, z_{m+1}\right]$ do not overlap, then:

$$
\begin{aligned}
J(r)= & {\left[\frac{\mathrm{e}^{k\left(\mathrm{i}\left(-\delta_{l}-1\right)+r\right) z_{l+1}}-\mathrm{e}^{k\left(\mathrm{i}\left(-\delta_{l}-1\right)+r\right) z_{l}}}{k\left(\mathrm{i}\left(-\delta_{l}-1\right)+r\right)}\right] } \\
& \cdot\left[\frac{\mathrm{e}^{k\left(\mathrm{i}\left(\delta_{m}+1\right)-r\right) z_{m+1}}-\mathrm{e}^{k\left(\mathrm{i}\left(\delta_{m}+1\right)-r\right) z_{m}}}{k\left(\mathrm{i}\left(\delta_{m}+1\right)-r\right)}\right]
\end{aligned}
$$

or

$$
\begin{aligned}
J(r)= & {\left[\frac{\mathrm{e}^{k\left(\mathrm{i}\left(\delta_{l}+1\right)-r\right) z_{l+1}}-\mathrm{e}^{k\left(\mathrm{i}\left(\delta_{l}+1\right)-r\right) z_{l}}}{k\left(\mathrm{i}\left(\delta_{l}+1\right)-r\right)}\right] } \\
& \cdot\left[\frac{\mathrm{e}^{k\left(\mathrm{i}\left(\delta_{m}-1\right)+r\right) z_{m+1}}-\mathrm{e}^{k\left(\mathrm{i}\left(\delta_{m}-1\right)+r\right) z_{m+1}}}{k\left(\mathrm{i}\left(\delta_{m}-1\right)+r\right)}\right] .
\end{aligned}
$$

In the case where $\left[z_{l}, z_{l+1}\right]$ and $\left[z_{m}, z_{m+1}\right]$ overlap, then Eq. (41) is split further into three integrals:

$$
\begin{aligned}
J(r) & =\int_{z_{l}}^{z_{m}} \int_{z_{m}}^{z_{l+1}} \mathrm{e}^{-r k(t-s)+\mathrm{i} k\left((t-s)+\delta_{m} t-\delta_{l} s\right)} \mathrm{d} t \mathrm{~d} s \\
& +\int_{z_{m}}^{z_{l+1}} \int_{z_{m}}^{z_{l+1}} \mathrm{e}^{-r k|s-t|+\mathrm{i} k\left((|s-t|)+\delta_{m} t-\delta_{l} s\right)} \mathrm{d} t \mathrm{~d} s \\
& +\int_{z_{l}}^{z_{l+1}} \int_{z_{l+1}}^{z_{m+1}} \mathrm{e}^{-r k(t-s)+\mathrm{i} k\left((t-s)+\delta_{m} t-\delta_{l} s\right)} \mathrm{d} t \mathrm{~d} s .
\end{aligned}
$$

Computing the first and third integral in Eq. (44) is a straightforward procedure and we can obtain similar formula as those of Eq. (42) or Eq. (43). The second integral of Eq. (44), is a total overlap of $\delta_{m}$ and $\delta_{l}$ (call it $J_{a}(r)$ ). Applying exponential power series to this integral leads to Eq. (45) (see top of the next page).

Now we are going to show how to evaluate the integral term:

$$
\int_{0}^{\infty} \frac{J(r)}{r^{\frac{1}{2}}(r-2 \mathrm{i})^{\frac{1}{2}}} \mathrm{~d} r
$$

This appears in Eq. (40). The term in the integral is singular when $r=0$. In order to remove this singularity, we first change the variables. Let $r=\frac{s^{2}}{1-s^{2}}$ then $\frac{d r}{d s}=\frac{2 s}{\left(1-s^{2}\right)^{2}}$, when $r=0$ implies $s=0$ and $r=\infty$ implies $s=1$.

$$
\begin{aligned}
& \int_{0}^{\infty} \frac{J(r)}{r^{\frac{1}{2}}(r-2 \mathrm{i})^{\frac{1}{2}}} \mathrm{~d} r= \\
& \quad \int_{0}^{1} \frac{J\left(\frac{s^{2}}{1-s^{2}}\right)}{\left(\frac{s^{2}}{1-s^{2}}\right)^{1 / 2}\left(\frac{s^{2}}{1-s^{2}}-2 \mathrm{i}\right)^{1 / 2}} \frac{2 s}{\left(1-s^{2}\right)^{2}} \mathrm{~d} s .
\end{aligned}
$$

Equation (47) appears to be singular at $s=1$ or $s=0$, but in fact it is not, since the function $J(r)$ in the numerator composed of exponential functions. Thus, it tends to zero faster then the denominator, as $s \rightarrow 1$ or $s \rightarrow 0$. We use a standard Gaussian quadrature rule to integrate Eq. (47).

Finally, when $\eta_{m}$ and $\eta_{l}$ are supported on different sides of the polygon $\left(\mathcal{K} \eta_{m}, \eta_{l}\right)$, they can only be evaluated numerically, since now $K(s, t)$ cannot be simplified further, which is why we have to use a standard Gaussian quadrature rule.

\subsection{Evaluation of $\left(F, \eta_{l}\right)$}

We shall now evaluate integrals of the form:

$$
\begin{aligned}
& \left(F, \eta_{l}\right)= \\
& \frac{1}{\sqrt{z_{l+1}-z_{l}}}[\underbrace{\int_{z_{l}}^{z_{l+1}}[2 f(s)-\Psi(s)] \mathrm{e}^{-\mathrm{i} k \delta_{l} s} \mathrm{~d} s}_{I_{1}} \\
& -\underbrace{2 \int_{z_{l}}^{z_{l+1}} \int_{0}^{L} K(s, t) \Psi(t) \mathrm{d} t \mathrm{e}^{-\mathrm{i} k \delta_{l} s} \mathrm{~d} s}_{I_{2}}] .
\end{aligned}
$$

Integral $I_{1}$ and some of $I_{2}$ can be evaluated analytically while some of $I_{2}$ must be computed numerically. When $\delta_{l}$ is supported on $\Gamma_{l}$, we need to consider two cases:

Case1 : If $l \leq n_{s h}$ then $\Gamma_{l}$ lies in the shadow region and $\Psi(s)=0$, hence $I_{2}=0$. Thus,

$$
\begin{aligned}
& I_{1}=\int_{z_{l}}^{z_{l+1}} 2 f(s) \mathrm{e}^{-\mathrm{i} k \delta_{l} s} \mathrm{~d} s= \\
& 2 \mathrm{e}^{\mathrm{i} k\left(g_{l} \sin \theta-h_{l} \cos \theta\right)} \\
& \cdot\left(\frac{\mathrm{e}^{\mathrm{i} k\left(\mathrm{e}_{l} \sin \theta-f_{l} \cos \theta-\delta_{l}\right) z_{l+1}}-\mathrm{e}^{\mathrm{i} k\left(\mathrm{e}_{l} \sin \theta-f_{l} \cos \theta-\delta_{l}\right) z_{l}}}{\mathrm{i} k\left(\mathrm{e}_{l} \sin \theta-f_{l} \cos \theta-\delta_{l}\right)}\right) .
\end{aligned}
$$

Case2 : If $l>n_{s h}$, then $\Gamma_{l}$ lies in the illuminated region, thus recalling Eqs. (12) and (15), we have:

$$
\begin{gathered}
I_{1}=\int_{z_{l}}^{z_{l+1}}[2 f(s)-\Psi(s)] \mathrm{e}^{-\mathrm{i} k \delta_{l} s} \mathrm{~d} s= \\
\int_{z_{l}}^{z_{l+1}} \frac{2 \beta}{\cos \theta^{\prime}+\beta} u^{i}(s) \mathrm{e}^{-\mathrm{i} k \delta_{l} s} \mathrm{~d} s= \\
\frac{2 \beta \mathrm{e}^{\mathrm{i} k\left(g_{l} \sin \theta-h_{l} \cos \theta\right)}}{\cos \theta^{\prime}+\beta} \\
\cdot\left(\frac{\mathrm{e}^{\mathrm{i} k\left(\mathrm{e}_{l} \sin \theta-f_{l} \cos \theta-\delta_{l}\right) z_{l+1}}-\mathrm{e}^{\mathrm{i} k\left(\mathrm{e} l \sin \theta-f_{l} \cos \theta-\delta_{l}\right) z_{l}}}{\mathrm{i} k\left(\mathrm{e}_{l} \sin \theta-f_{l} \cos \theta-\delta_{l}\right)}\right) .
\end{gathered}
$$




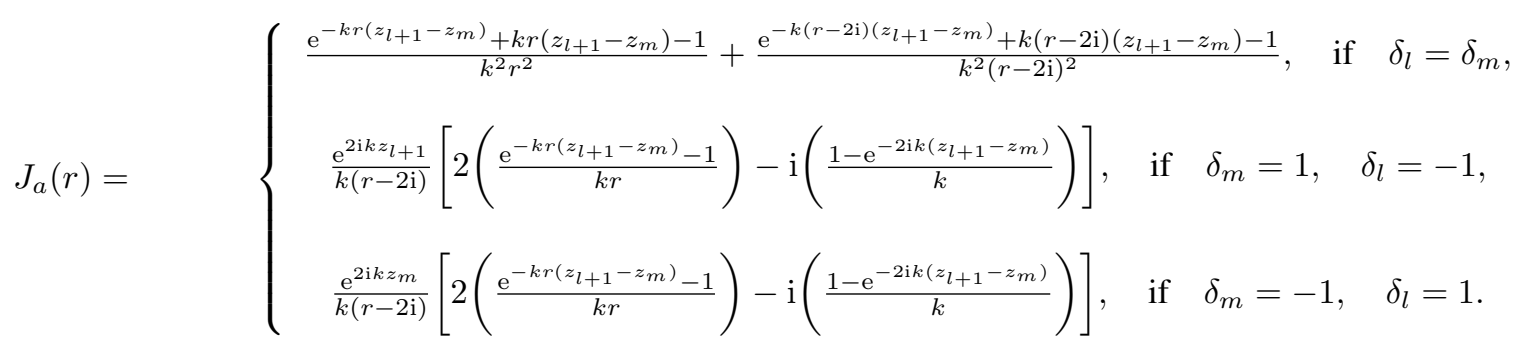

$$
\begin{gathered}
I_{2}=2 \int_{z_{l}}^{z_{l+1}}\left[\int_{\sum_{m=1}^{n_{s h} L_{m}}}^{L} K(s, t) \Psi(t) \mathrm{d} t\right] \mathrm{e}^{-\mathrm{i} k \delta_{l} s} \mathrm{~d} s= \\
2 \sum_{m=n_{s h}+1}^{n_{s}} \int_{\sum_{p=1}^{m-1} L_{p}}^{\sum_{p=1}^{m} L_{p}}\left[\int_{z_{l}}^{z_{l+1}} K(s, t) \mathrm{e}^{-\mathrm{i} k \delta_{l} s} \mathrm{~d} s\right] \Psi(t) \mathrm{d} t= \\
\frac{4 \cos \theta^{\prime}}{\cos \theta^{\prime}+\beta} \\
\sum_{m=n_{s h}+1}^{n_{s}} \int_{\sum_{p=1}^{m-1} L_{p}}^{\sum_{p=1}^{m} L_{p}}\left[\int_{z_{l}}^{z_{l+1}} K(s, t) \mathrm{e}^{-\mathrm{i} k \delta_{l} s} \mathrm{~d} s\right] u^{i}(t) \mathrm{d} t= \\
\frac{4 \cos \theta^{\prime}}{\cos \theta^{\prime}+\beta} \sum_{m=n_{s h}+1}^{n_{s}} \mathrm{e}^{\left.\mathrm{i} k\left(g_{m} \sin \theta-h_{m} \cos \theta\right)\right]} \\
\cdot\left[\int_{\sum_{p=1}^{m-1} L_{p}}^{\sum_{p=1}^{m} L_{p}} \int_{z_{l}}^{z_{l+1}} K(s, t)\right. \\
\frac{4 \cos \theta^{\prime}}{\cos \theta^{\prime}+\beta} \sum_{m=n_{s h a d}+1}^{n} \mathrm{e}^{\mathrm{i} k\left(g_{m} \sin \theta-h_{m} \cos \theta\right)} I 2_{l, m} . \quad(51)
\end{gathered}
$$

We now evaluate $I 2_{l, m}$ when $s$ and $t$ are supported on the same side of the polygon. Recalling Eq. (38),

$$
\begin{aligned}
& I 2_{l, m}= \\
& \int_{\sum_{p=1}^{m-1} L_{p}}^{\sum_{p=1}^{m} L_{p}} \int_{z_{l}}^{z_{l+1}} K(s, t) \mathrm{e}^{\mathrm{i} k\left(\left(\mathrm{e}_{m} \sin \theta-f_{m} \cos \theta\right) t-\delta_{l} s\right)} \mathrm{d} t \mathrm{~d} s=
\end{aligned}
$$$$
\frac{-k \beta}{4} \int_{\sum_{p=1}^{m-1} L_{p}}^{\sum_{p=1}^{m} L_{p}} \int_{z_{l}}^{z_{l+1}} H_{0}^{(1)}(k|s-t|)
$$$$
\cdot \mathrm{e}^{\mathrm{i} k\left(\left(e_{m} \sin \theta-f_{m} \cos \theta\right) t-\delta_{l} s\right)} \mathrm{d} t \mathrm{~d} s=
$$$$
\frac{\mathrm{i} k \beta}{2 \pi} \int_{0}^{\infty} \frac{I^{*}(r)}{r^{1 / 2}(r-2 \mathrm{i})^{1 / 2}} \mathrm{~d} r
$$

where

$$
I^{*}(r)=\int_{z_{m}}^{z_{m+1}} \int_{z_{l}}^{z_{l+1}} \mathrm{e}^{(\mathrm{i}-r) k|s-t|+\mathrm{i} k\left(\delta_{m} t-\delta_{l} s\right)} \mathrm{d} t \mathrm{~d} s ;
$$

where $z_{m}=\sum_{p=1}^{m-1} L_{p}, z_{m+1}=\sum_{p=1}^{m} L_{p}, \delta_{m}=e_{m} \sin \theta-$ $f_{m} \cos \theta$. Evaluating Eq. (53) (with a little more effort) leads to Eq. (54) (see top of the next page).

Finally, when $s$ and $t$ are on different side of polygon, we again use the standard Gaussian quadrature rule.

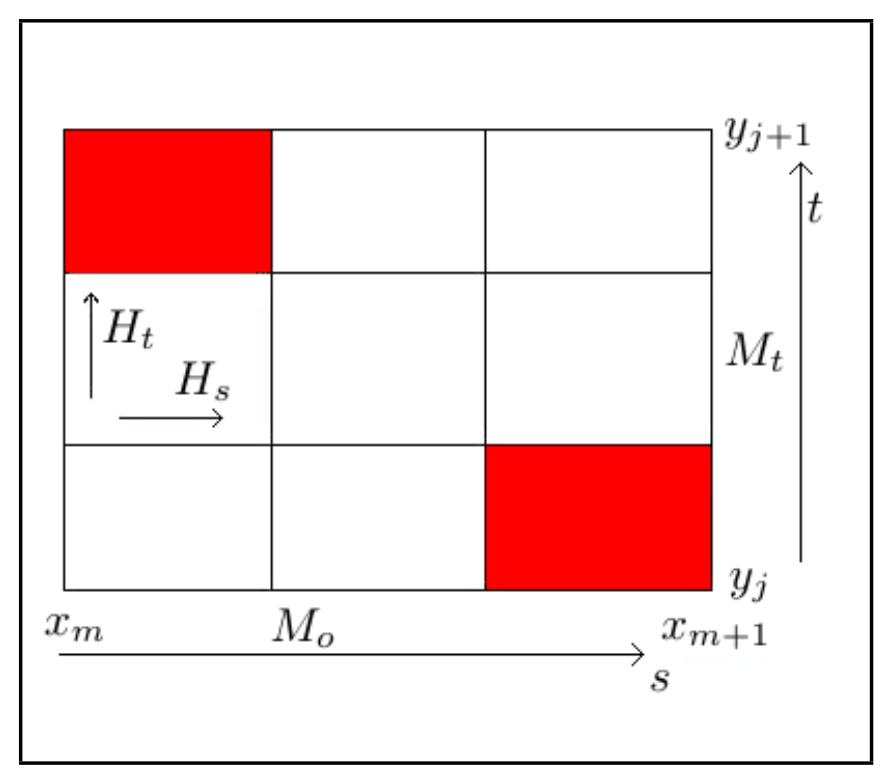

Figure 2. The integrating rectangle.

\section{NUMERICAL EVALUATION OF NON-OVERLAPPING INTEGRALS}

To evaluate non-overlapping integrals, we first divide the big rectangle $\left[t_{m}, t_{m+1}\right] \times\left[t_{j}, t_{j+1}\right]$ into equally spaced small rectangles with each of sides $H_{s} \times H_{t}$, as shown in Fig.2.

In Fig. $2, M_{s}$ are nodes length between $\left[t_{m}, t_{m+1}\right]$ in the direction $s$ and $M_{t}$ are nodes length between $\left[t_{j}, t_{j+1}\right]$ in the direction $t$. Now define

$$
H_{s}:=\frac{t_{m+1}-t_{m}}{M_{s}}, \quad H_{t}:=\frac{t_{j+1}-t_{j}}{M_{t}} .
$$

To deal with the oscillatory nature of integrand, we chose $M_{s} \propto k$ and $M_{t} \propto k$. More specifically, we chose $H_{t} \sim 1 / k$ and $H_{s} \sim 1 / k$, then used the two-dimensional Gaussian quadrature rule to approximate each integral on each small rectangle as follows:

$$
\begin{gathered}
\int_{t_{m}}^{t_{m}+1} \int_{t_{j}}^{t_{j+1}} K(s, t) \mathrm{e}^{\mathrm{i} k\left(\sigma_{j} t-\sigma_{m} s\right)} \mathrm{d} t \mathrm{~d} s= \\
\sum_{m=1}^{M_{s}} \sum_{j=1}^{M_{t}} \int_{t_{m}+(m-1) H_{s}}^{t_{m}+m H_{s}} \int_{t_{j}+(j-1) H_{t}}^{t_{j}+j H_{t}} K(s, t) \mathrm{e}^{\mathrm{i} k\left(\sigma_{j} t-\sigma_{m} s\right)} \mathrm{d} t \mathrm{~d} s= \\
\sum_{m=1}^{M_{s}} \sum_{j=1}^{M_{t}} \sum_{l=1}^{m_{s}} \sum_{n=1}^{m_{t}} w_{l} w_{n} K\left(s_{l}, t_{n}\right) \mathrm{e}^{\mathrm{i} k\left(\sigma_{j} t_{n}-\sigma_{m} s_{l}\right)}
\end{gathered}
$$




$$
\begin{aligned}
& I^{*}(r)=\mathrm{e}^{\mathrm{i} k\left(\delta_{m}-1\right) z_{m}}\left[\frac{\mathrm{e}^{\mathrm{i} k\left(1-\delta_{l}\right) z_{l+1}+k r\left(z_{m}-z_{l+1}\right)}-\mathrm{e}^{\mathrm{i} k\left(1-\delta_{l}\right) z_{l}+k r\left(z_{m}-z_{l}\right)}}{k\left(r-\mathrm{i}\left(1-\delta_{m}\right) k\left(r-\mathrm{i}\left(1-\delta_{l}\right)\right)\right.}\right] \\
& +\mathrm{e}^{\mathrm{i} k\left(\delta_{m}+1\right) z_{m+1}}\left[\frac{\mathrm{e}^{-\mathrm{i} k\left(1+\delta_{l}\right) z_{l+1}+k r\left(z_{l}-z_{m+1}\right)}-\mathrm{e}^{-\mathrm{i} k\left(1+\delta_{l}\right) z_{l+1}+k r\left(z_{l+1}-z_{m+1}\right)}}{k\left(r-\mathrm{i}\left(1+\delta_{m}\right) k\left(r-\mathrm{i}\left(1+\delta_{l}\right)\right)\right.}\right] \\
& +2 \frac{\left(\mathrm{e}^{\mathrm{i} k\left(\delta_{m}-\delta_{l}\right) z_{l+1}}-\mathrm{e}^{\mathrm{i} k\left(\delta_{m}-\delta_{l}\right) z_{l}}\right)}{\mathrm{i} k^{2}\left(\delta_{m}-\delta_{l}\right)}\left[\frac{r-\mathrm{i}}{\delta_{m}^{2}+(r-\mathrm{i})^{2}}\right] .
\end{aligned}
$$

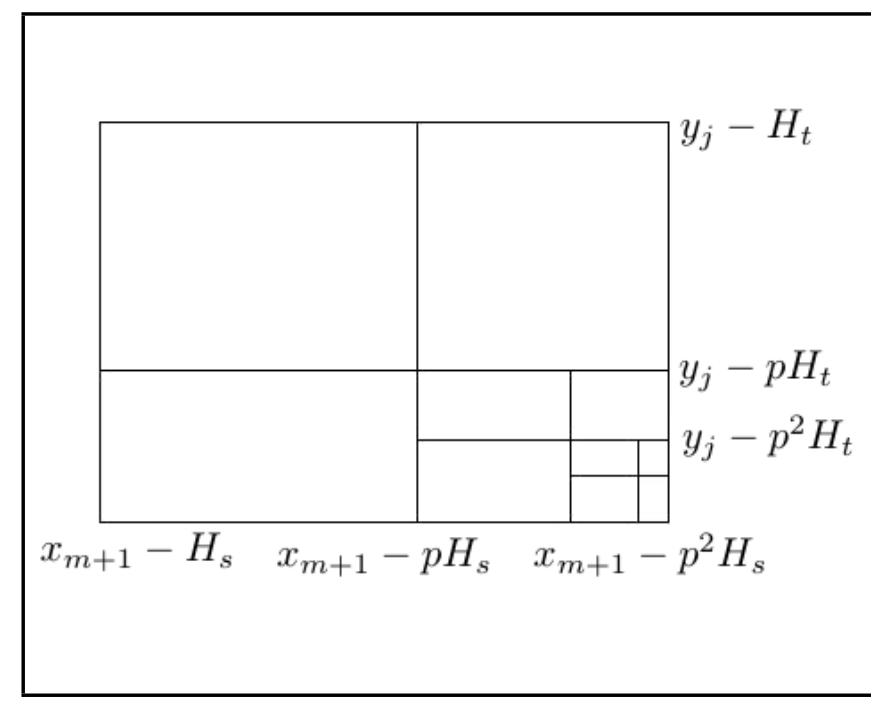

Figure 3. Removing the singularity.

where $m_{s}$ and $m_{t}$ are the number of Gaussian points between $\left[t_{m}+(m-1) H_{s}, t_{m}+m H_{s}\right]$ and $\left[t_{j}+(j-1) H_{t}, t_{j}+j H_{t}\right]$ respectively, and $w_{l}$ and $w_{n}$ are weights. We will, however, still have a singularity when the supports of $\eta_{j}$ and $\eta_{l}$ touch or are close to each other. This situation can occur at the corners of the shaded region shown in Fig.2, that is, when either $t_{m+1}-t_{j} \leq \epsilon$ or $t_{j+1}-t_{m} \leq \epsilon$, where $\epsilon>0$ is a small number close or equal to zero. To deal with this situation, we take the shaded rectangle $\left[t_{m+1}-H_{s}, t_{m+1}\right] \times\left[t_{j}, t_{j}+H_{t}\right]$ (the lower bottom) in Fig.2 and subdivide it into small rectangles, with the nodes highly concentrated near the peaked area as shown in Fig.3. We place the nodes at $t_{m+1}-p^{l} H_{s}, l=0, \ldots, n$ ( $n$ is the number of Gaussian quadrature points and $p=0.15$ ) on $\left[t_{m+1}-H_{s}, t_{m+1}\right]$ and at $t_{j}+p^{l} H_{t}, l=0, \ldots, n$ on $\left[t_{j}, t_{j}+H_{t}\right]$. We again use the two-dimensional Gaussian quadrature rule to approximate each integral on each small rectangle, thus arriving at an equation similar to Eq. (56).

\section{NUMERICAL RESULTS}

As our numerical example, we take the scattering object $\Omega$, to be a square, with vertices $(0,0),(2 \pi, 0),(2 \pi, 2 \pi)$, and $(0,2 \pi)$. We take $\beta=1$ on each side $\Gamma_{j}$ and the incident angle $\theta=\pi / 4$ so that the plane wave is directed towards the corner at $(0,2 \pi)$. The reflective angle in Eq. (15) is given by

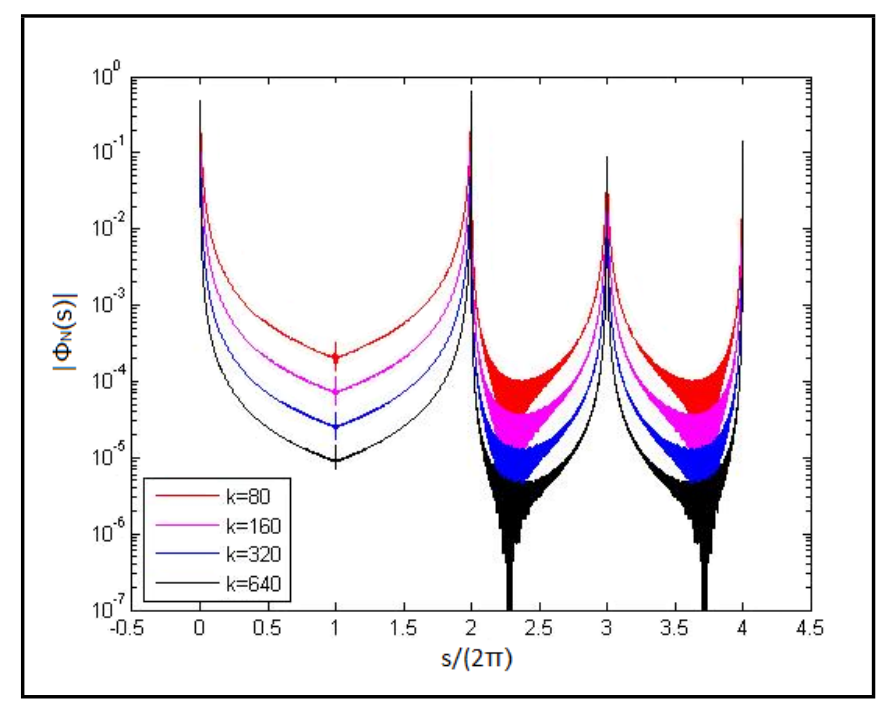

Figure 4. Behaviour of the solution for increasing $k$, when $N_{1}=128$.

$\theta^{\prime}=\pi / 4$ and $-\pi / 4$ in the illuminated regions $\Gamma_{3}$ and $\Gamma_{4}$ respectively. We code the system Eq. (32) to get the unknown $v_{m}$ and use Eq. (27) to get $\Phi_{N}$. For this particular example, we chose the mesh such that for a fixed $N_{1}$, we use Eq. (21) to compute $N$, and we use Eq. (20) to compute $N_{L_{j}, \lambda, q_{j}}$. In Fig. 4 we plot a comparison of the solutions for $N_{1}=128$ for $k=80,160,320$, and 640 . As $k$ increases, the diffracted wave decays away faster from the corners. Table 1 shows the errors for fixed $N_{1}=32$ and increasing $k$. The results in Table 1 shows that errors are inversely proportional to $k$ that is for increasing $k$ the error decreases. This effect is reflected in column $\left\|\Phi_{128}-\Phi_{N_{1}}\right\|_{2}$ in Table 1 . The relative $L^{2}$ errors $\left\|\Phi_{128}-\Phi_{N_{1}}\right\|_{2} /\left\|\Phi_{128}\right\|_{2}$ remain relatively constant as $k$ increases, which is a good sign for the robustness of our scheme. The degrees of freedom $D_{N_{1}}$ is proportional to $\log (k)$, that is, $D_{N_{1}}$ increase logarithmically as the wavenumber increases.

\section{CONCLUSIONS}

In this paper, we have described how to implement the boundary element method developed in ${ }^{4}$ for high frequency scattering by convex polygons with impedance boundary conditions. We explaine in detail how to solve the resulting system of linear equation. We encounter an integration scheme that is frequency independent when we are integrating over elements 
Table 1. Relative errors, scattering by square, $N_{1}=32$.

\begin{tabular}{||c|c|c|c||}
\hline $\mathrm{k}$ & $D_{N_{1}}$ & $\left\|\Phi_{128}-\Phi_{32}\right\|_{2}$ & $\left\|\Phi_{128}-\Phi_{32}\right\|_{2} /\left\|\Phi_{128}\right\|_{2}$ \\
\hline 5 & 376 & $2.1229 \times 10^{-2}$ & $4.9412 \times 10^{-2}$ \\
\hline 10 & 464 & $1.4873 \times 10^{-2}$ & $4.9022 \times 10^{-2}$ \\
\hline 20 & 552 & $1.0520 \times 10^{-2}$ & $4.9006 \times 10^{-2}$ \\
\hline 40 & 640 & $7.3863 \times 10^{-3}$ & $4.8627 \times 10^{-2}$ \\
\hline 80 & 728 & $5.3551 \times 10^{-3}$ & $4.9871 \times 10^{-2}$ \\
\hline 160 & 816 & $3.6531 \times 10^{-3}$ & $4.8177 \times 10^{-2}$ \\
\hline 320 & 904 & $2.4775 \times 10^{-3}$ & $4.6072 \times 10^{-2}$ \\
\hline
\end{tabular}

supported on the same side of the polygon, but our scheme for elements supported on different sides of the polygon has a complicated cost that grows with frequency. For this particular problem, we apply the standard Gaussian quadrature rule in our code to solve such integrals. For our future work, it will be interesting to apply frequency independent schemes for these integrals, such as the ones that have been developed recently by Melenk and Langdon ${ }^{15}$ for the problem of scattering by sound soft convex polygons.

\section{REFERENCES}

1 Perrey-Debain, E., Laghrouche, O., Bettess, P., and Trevelyan, J. Plane-wave basis finite elements and boundary elements for three-dimensional wave scattering. Philosophical Transactions of the Royal Society of London A: Mathematical, Physical and Engineering Sciences, 362(1816), 561-577, (2004). http://dx.doi.org/10.1098/rsta.2003.1335.

2 Perrey-Debain, E., Trevelyan, J., and Bettess, P. Plane wave interpolation in direct collocation boundary element method for radiation and wave scattering: numerical aspects and applications. Journal of sound and vibration, 261(5), 839-858, (2003). http://dx.doi.org/10.1016/S0022460X(02)01006-4.

3 Chandler-Wilde, S. N., Graham, I. G., Langdon, S., and Spence, E. A. Numerical-asymptotic boundary integral methods in high frewuency acoustic scattering. Acta numerica, 21, 89-305, (2012). http://dx.doi.org/10.1017/S0962492912000037.

4 Chandler-Wilde, S., Langdon, S., and Mokgolele, M. A high frequency boundary element method for scattering by convex polygons with impedance boundary conditions. Communications in Computational Physics, 11(02), 573-593, (2012). http://dx.doi.org/10.4208/cicp.231209.040111s.
5 Mokgolele, M. Numerical solution of high frequency acoustic scattering problems. PhD Thesis, University of Reading, UK, , (2009).

6 McLean, W. C. H. Strongly elliptic systems and boundary integral equations. Cambridge university press, (2000).

7 Colton, D. and Kress, R. Inverse acoustic and electromagnetic scattering theory, volume 93. Springer Science \& Business Media, (2012). http://dx.doi.org/10.1007/978-3662-03537-5.

8 Schenck, H. A. Improved integral formulation for acoustic radiation problems. The journal of the acoustical society of America, 44(1), 41-58, (1968). http://dx.doi.org/10.1121/1.1911085.

9 Chandler-Wilde, S. N. and Langdon, S. A galerkin boundary element method for high frequency scattering by convex polygons. SIAM Journal on Numerical Analysis, 45(2), 610-640, (2007). http://dx.doi.org/10.1137/06065595X.

10 Chandler-Wilde, S., Langdon, S., and Ritter, L. A high-wavenumber boundary-element method for an acoustic scattering problem. Philosophical Transactions of the Royal Society of London A: Mathematical, Physical and Engineering Sciences, 362(1816), 647-671, (2004). http://dx.doi.org/10.1098/rsta.2003.1339.

11 Langdon, S. and Chandler-Wilde, S. Implementation of a boundary element method for high frequency scattering by convex polygons. Advances in Boundary Integral Methods (Proceedings of the 5th UK Conference on Boundary Integral Methods), , 2-11, (2005).

12 Huybrechs, D. and Vandewalle, S. On the evaluation of highly oscillatory integrals by analytic continuation. Katholieke Universiteit Leuven, Report TW431, , (2005).

13 Huybrechs, D. and Vandewalle, S. The efficient evaluation of highly oscillatory integrals in bem by analytic continuation. Advances in Boundary Integral Methods, (Proceedings of the 5th UK Conference on Boundary Integral Methods), , 12-21, (2005).

14 Abramowitz, M., Stegun, I. A., et al. Handbook of mathematical functions. Applied mathematics series, 55, 62, (1966).

15 Melenk, J. M. and Langdon, S. An hp-bem for high frequency scattering by convex polygons. Proc. 8th Int. Conf. on Mathematical and Numerical Aspects of Wave Propagation, Reading University, , 93-95, (2007). 